\title{
A QSAT Benchmark Based on Vertex-Folkman Problems (Student Abstract)
}

\author{
David E. Narváez \\ Golisano College of Computing and Information Science \\ Rochester Institute of Technology \\ Rochester, NY 14620 \\ den9562@rit.edu
}

\begin{abstract}
The purpose of this paper is to draw attention to a particular family of quantified Boolean formulas (QBFs) stemming from encodings of some vertex Folkman problems in extremal graph theory. We argue that this family of formulas is interesting for QSAT research because it is both conceptually simple and parametrized in a way that allows for a finegrained diversity in the level of difficulty of its instances. Additionally, when coupled with symmetry breaking, the formulas in this family exhibit backbones (unique satisfying assignments) at the top-level existential variables. This benchmark is thus suitable for addressing questions regarding the connection between the existence of backbones and the hardness of QBFs.
\end{abstract}

\section{Introduction}

Graph theory has served as an excellent source of problems where SAT techniques can be evaluated against (Urquhart 1987; Krishnamurthy and Moll 1981; Järvisalo et al. 2012). The particular graph problems we look at in this paper are vertex Folkman problems (Folkman 1970) that consider the existence of graphs that do not contain cliques of a given size and are such that their vertices cannot be colored in a way that avoids cliques of prescribed orders in each color. In Folkman theory, a theorem by Łuczak, Ruciński, and Urbański (2001) guarantees that the instances obtained from a particular choice of parameters have unique solutions. When these instances are interpreted as QBFs, and under appropriate symmetry breaking, this theorem guarantees the corresponding formulas have unique satisfying assignments to a subset of the top-level existential variables. This behavior, typically referred to as a backbone in the SAT research community (Williams, Gomes, and Selman 2003; Kilby et al. 2005), has been linked to the hardness of SAT instances but, to the best of our knowledge, has not yet been formally studied in the context of QSAT.

\section{Background}

Let $K_{n}$ be the complete graph on $n$ vertices. We define the set of Folkman graphs $\mathcal{F}^{N}\left(a_{1}, a_{2}, \ldots, a_{r} ; m\right)$, where $N$ and

Copyright (c) 2020, Association for the Advancement of Artificial Intelligence (www.aaai.org). All rights reserved. $m$ are natural numbers and $\left(a_{c}\right)_{1 \leq c \leq r}$ is a sequence of natural numbers such that $a_{c} \geq 2$, as the set of all graphs $G$ of order $N$ that are $K_{m}$-free and are such that for every coloring of the vertices of $G$ using $r$ colors (hereon called $r$-colorings of the vertices of $G$ ) there exists a color $c \in\{1,2, \ldots, r\}$ such that there is a monochromatic $K_{a_{c}}$ in color $c$.

Consider the problem of determining whether $\mathcal{F}^{N}\left(a_{1}, a_{2}, \ldots, a_{r} ; m\right)$ is non-empty. It is possible to encode this problem as a $\mathrm{QBF}$ using $\left(\begin{array}{c}N \\ 2\end{array}\right)$ variables $x_{i, j}$ and $r N$ variables $y_{i, c}$ such that the formula is satisfiable if and only if $\mathcal{F}^{N}\left(a_{1}, a_{2}, \ldots, a_{r} ; m\right)$ is non-empty. The variable $x_{i, j}$ is set to true if and only if there exists an edge between vertices $i$ and $j$, while the variable $y_{i, c}$ is set to true if and only if vertex $i$ has color $c$. The intuition behind our encoding, which we present next, is that we are looking for a graph $G$ that avoids $K_{m}$ as a subgraph and is such that for all valid assignments to the variables $y_{i, c}$ there exists a $c \in\{1,2, \ldots, r\}$ and a subset $T \subseteq\{1,2, \ldots, N\}$ of cardinality $a_{c}$ such that all $x_{i, j}$ for $i, j \in T, i \neq j$, and all $y_{i, c}$ for $i \in T$ are true. To avoid $K_{m}$ as a subgraph, the formula

$$
A_{m}^{N}=\bigwedge_{\substack{S \subseteq\{1,2, \ldots, N\} \\|S|=m}} \bigvee_{\substack{i, j \in S, i \neq j}} \overline{x_{i, j}}
$$

guarantees that for no subset $S \subseteq\{1,2, \ldots, N\}$ of cardinality $m$ are the variables $x_{i, j}$ all true for $i, j \in S$ with $i \neq j$. In order to consider only valid $r$-colorings, the formula

$$
U_{r}^{N}=\bigwedge_{i=1}^{N}\left(\left(\bigvee_{c=1}^{r} y_{i, c}\right) \wedge\left(\bigwedge_{\substack{c, d \in\{1, \ldots, r\} \\ c \neq d}}\left(\overline{y_{i, c}} \vee \overline{y_{i, d}}\right)\right)\right)
$$

guarantees that exactly one of $y_{i, 1}, y_{i, 2}, \ldots, y_{i, r}$ is true for each vertex $i$. The formula

$$
C_{n, c}^{N}=\bigvee_{\substack{T \subseteq\{1,2, \ldots, N\} \\|T|=n}}\left(\left(\bigwedge_{\substack{i, j \in T \\ i \neq j}} x_{i, j}\right) \wedge\left(\bigwedge_{i \in T} y_{i, c}\right)\right)
$$

is true if there is a subset $T \subseteq\{1,2, \ldots, N\}$ of cardinality $n$ such that all the edges between these vertices exist in $G$ (i.e., $x_{i, j}$ is true for all $i, j \in T, i \neq j$ ) and all the vertices in $T$ 
are of the same color $c$. Putting these together, the formula

$$
\begin{aligned}
F^{N}\left(a_{1}, \ldots, a_{r} ; m\right)= & \exists\left(x_{i, j}\right)_{1 \leq i<j \leq N} \\
& \forall\left(y_{i, c}\right)_{1 \leq i \leq N, 1 \leq c \leq r} \\
& A_{m}^{N} \wedge\left(U_{r}^{N} \rightarrow \bigvee_{c=1}^{r} C_{a_{c}, c}^{N}\right)(1)
\end{aligned}
$$

is true if and only if $\mathcal{F}^{N}\left(a_{1}, a_{2}, \ldots, a_{r} ; m\right)$ is non-empty.

For the rest of the paper, we set $a=\max \left\{a_{1}, a_{2}, \ldots, a_{r}\right\}$. Łuczak, Ruciński, and Urbański (2001) proved the following.

Theorem 1 ((Luczak, Ruciński, and Urbański 2001)) For every non-decreasing sequence of natural numbers $a_{1}, a_{2}, \ldots, a_{r}, a_{c} \geq 2$, and $m=1+\sum_{c=1}^{r}\left(a_{c}-1\right)$ the graph $K_{a_{r}+m}-C_{2 a_{r}+1}$ is the unique graph in $\mathcal{F}^{a_{r}+m}\left(a_{1}, a_{2}, \ldots, a_{r} ; m\right)$.

From Theorem 1 we can derive several properties of Formula (1) for these parameters. By counting the number of cycles of length $2 a+1$ in $K_{m+a}$, we can see that $F^{m+a}\left(a_{1}, a_{2}, \ldots, a_{r} ; m\right)$ has exactly $\left(\begin{array}{c}m+a \\ 2 a+1\end{array}\right) \frac{(2 a) !}{2}$ satisfying assignments of the $x_{i, j}$ variables, so in particular it is satisfiable. It is also evident that all graphs represented by these satisfying assignments are pairwise isomorphic. This can be exploited to create a family of Boolean formulas with a unique satisfying assignment for the top-level existential variables. In the following section we explore how symmetry breaking can be used for this purpose.

\subsection{Symmetry Breaking}

Recall that two graphs $G$ and $H$ are isomorphic if they are essentially the same up to a permutation of their vertices. The set of graphs of order $n$ can then be partitioned into equivalence classes $\mathcal{I}_{n}$ such that for each class $C \in \mathcal{I}_{n}$, all graphs in $C$ are pairwise isomorphic. A symmetry breaking technique for generic graph-search problems is perfect if exactly one graph from each class in $\mathcal{I}_{n}$ is admitted. We can obtain a family of QBF benchmarks based on Theorem 1, each having a unique solution by using partial symmetry breaking. The partial symmetry breaking strategy we explore in this paper is based on the observation that it is sufficient to have perfect symmetry breaking for the subset $\mathcal{I}_{m+a}^{\leq 2 a+1} \subset \mathcal{I}_{m+a}$ of isomorphism classes of graphs of order $m+a$ with at least $\left(\begin{array}{c}m+a \\ 2\end{array}\right)-(2 a+1)$ edges. In this case, one could add the constraint

$$
\operatorname{AtMostK}\left(\left(\overline{x_{u, v}}\right)_{u, v<a+m, u \neq v} ; 2 a+1\right) \rightarrow \operatorname{SB}\left(\mathcal{I}_{m+a}^{\leq 2 a+1}\right)
$$

and $\operatorname{SB}\left(\mathcal{I}_{m+a}^{\leq 2 a+1}\right)$ can be built from enumerating all nonisomorphic graphs with at least $\left(\begin{array}{c}m+a \\ 2\end{array}\right)-(2 a+1)$ edges through the nauty (McKay and Piperno 2014) package and then building a sum-of-products predicate from the edge variables involved.

\section{Implementation and Experiments}

We implemented software to generate instances from our benchmark in QDIMACS ${ }^{1}$ and QCIR-14 (Jordan, Klieber,

\footnotetext{
${ }^{1}$ http://www.qbflib.org/qdimacs.html
}

and Seidl 2016) formats, thus covering the majority of QSAT solvers available. We also implemented model enumerators for instances of this benchmark. These enumerators verify the correctness of the instances generated since they enumerate the unique solution modulo symmetries. The software and sample running times of some solvers on these instances are available online ${ }^{2}$.

\section{Future Work}

A natural next step would be to complement this work with a theoretical analysis that could shed light on better solving algorithms or heuristics that target this family of formulas in the hope that generalizations of some of these techniques could help QSAT solvers at large. It would be interesting to determine whether there are short proofs for the instances in our benchmark when using proof systems that include symmetry rules (Kauers and Seidl 2018; Blinkhorn and Beyersdorff 2019). Such proofs would provide concrete performance measures as the length of proofs provided by the current QSAT solvers could be compared to the ones in proof systems with symmetry rules.

\section{Acknowledgments}

The author would like to thank Edith Hemaspaandra, Stanisław Radziszowski, and the anonymous AAAI reviewers for their valuable comments. The author was supported in part by NSF-DUE-1819546.

\section{References}

Blinkhorn, J., and Beyersdorff, O. 2019. Proof complexity of QBF symmetry recomputation. In SAT, volume 11628 of LNCS, 36-52. Springer.

Folkman, J. 1970. Graphs with monochromatic complete subgraphs in every edge coloring. SIAM Journal on Applied Mathematics 18(1):19-24.

Järvisalo, M.; Matsliah, A.; Nordström, J.; and Živnỳ, S. 2012. Relating proof complexity measures and practical hardness of SAT. In $C P, 316-331$. Springer.

Jordan, C.; Klieber, W.; and Seidl, M. 2016. Non-CNF QBF solving with QCIR. In Darwiche, A., ed., AAAI Workshop: Beyond NP, volume WS-16-05 of AAAI Workshops. AAAI Press.

Kauers, M., and Seidl, M. 2018. Short proofs for some symmetric quantified Boolean formulas. IPL 140:4-7.

Kilby, P.; Slaney, J.; Thiébaux, S.; and Walsh, T. 2005. Backbones and backdoors in satisfiability. In AAAI, 1368.

Krishnamurthy, B., and Moll, R. N. 1981. Examples of hard tautologies in the propositional calculus. In STOC, 28-37.

Łuczak, T.; Ruciński, A.; and Urbański, S. 2001. On minimal Folkman graphs. Discrete Mathematics 236(1-3):245-262.

McKay, B. D., and Piperno, A. 2014. Practical graph isomorphism, II. Journal of Symbolic Computation 60:94-112.

Urquhart, A. 1987. Hard examples for resolution. JACM 34(1):209-219.

Williams, R.; Gomes, C. P.; and Selman, B. 2003. Backdoors to typical case complexity. In IJCAI, volume 3, 1173-1178.

\footnotetext{
${ }^{2}$ https://doi.org/10.5281/zenodo.3548977
} 